\title{
INIENCEFALIA: PRIMER CASO REPORTADO EN COLOMBIA Y REVISIÓN DE LA LITERATURA
}

\author{
Iniencephaly: first case reported in Colombia and \\ literature review \\ Wilmar Saldarriaga-Gil, M.D., M.Sc. *, Fabián Andrés Ruiz-Murcia**, Carolina \\ Isaza, M.D., M.Sc. *** \\ Recibido: mayo 2/11 - Aceptado: diciembre 16/11
}

\section{RESUMEN}

Introducción: la iniencefalia es un defecto del tubo neural, de baja frecuencia, del cual no hay valores de prevalencia establecidos. Esta patología se caracteriza por un ensanchamiento del foramen magno, raquisquisis y retroflexión marcada de la cabeza. El objetivo de este artículo es reportar un caso con los hallazgos clásicos de iniencefalia, y revisar la literatura.

Materiales y métodos: se presenta el caso de un recién nacido de sexo femenino, producto de madre primigestante de 14 años, que consultó a institución de tercer nivel de complejidad, que atiende pacientes en su mayoría de la red pública del suroccidente colombiano, con ecografía prenatal que mostró feto con defecto a nivel de vértebras cervicales y torácicas. Al recién nacido se le realizó autopsia y radiografías, en los que se evidenciaron anomalías clásicas de iniencefalia.

\footnotetext{
* Médico, Especialista en Ginecología y Obstetricia. Maestría en Ciencias Básicas Médicas, Énfasis en Embriología y Genética. MACOS Grupo de Malformaciones Congénitas Perinatales y Dismorfología, Universidad del Valle. Profesor Asociado. Departamentos de Morfología, Ginecología y Obstetricia, Facultad de Salud, Universidad del Valle. Hospital Universitario del Valle, ESE Ladera. Cali (Colombia). Correo electrónico: HYPERLINK “mailto:wsaldarriaga0608@yahoo.com” wsaldarriaga0608@yahoo.com

** Estudiante Cuarto Año, Pregrado de Medicina y Cirugía, Universidad del Valle. Cali (Colombia).

*** Médico, Maestría en Ciencias Básicas Médicas, Énfasis Embriología y Genética. MACOS Grupo de Malformaciones Congénitas Perinatales y Dismorfología, Universidad del Valle. Profesora Titular. Departamento de Morfología, Facultad de Salud, Universidad del Valle. Vicerrectora de Investigaciones, Universidad del Valle. Cali (Colombia).
}

Se realiza búsqueda en las base de datos Medline vía PubMed por medio de la palabra clave iniencephaly.

Conclusión: la importancia de este reporte radica en sumar a la literatura un nuevo caso de iniencefalia asociado con meningocele e hipoplasia de radio y ulna, adicionalmente es el primero reportado en Colombia. Palabras clave: iniencefalia, defectos del tubo neural, ECLAMC.

\section{SUMMARY}

Introduction: iniencephaly is a rarely occurring defect of the neural tube, lacking established prevalence values to date. It is characterized by widening of the foramen magnum, rachischisis and marked retroflexion of the head. This article was aimed at reporting a case having classical findings of iniencephaly and providing a literature review. Materials and methods: the case of a newly born female is presented; her first-time mother was aged 14 and consulted a third level hospital mainly attending patients from the southwestern Colombian public healthcare network. The prenatal echography showed a fetus having a cervical/ thoracic vertebrae defect. The newborn was subjected to autopsy and radiographies, revealing classical anomalies regarding iniencephaly. A search was made of Medline databases via PubMed using the key word "iniencephaly".

Conclusion: this report's importance lies in adding a new case of iniencephaly associated with 
meningocele and hypoplasia of the radius and ulna to the pertinent literature. It is the first case reported in Colombia.

Key words: iniencephaly, neural tube defect, LatinAmerican Congenital Malformation Collaborative Study (Estudio Colaborativo Latinoamericano de Malformaciones Congénitas - ECLAMC).

\section{INTRODUCCIÓN}

La iniencefalia es un defecto del tubo neural, poco frecuente y extremadamente grave, usualmente incompatible con la vida, ${ }^{1,2}$ del cual no se conocen valores precisos de prevalencia, y solo existen reportes de series o casos aislados. ${ }^{1,3}$ Fue descrito por primera vez en Francia por Saint-Hilaire en 1836 y se caracteriza por la presencia de un defecto en la porción escamosa del hueso occipital que lleva a un ensanchamiento del foramen magno, vértebras cervicales defectuosas con disrrafismo (raquisquisis) y retroflexión marcada de la cabeza., Estos defectos sumados llevan a que la cabeza esté permanentemente unida a la espalda y produzca continuidad de tejidos blandos subyacentes y el defecto quede total o parcialmente recubierto por piel. ${ }^{1,3}$ Su nombre se origina de las palabras griegas inion (cuello) y céfalo (cabeza). ${ }^{4}$

El objetivo de este manuscrito es presentar un caso de iniencefalia, asociado con meningocele e hipoplasia de radio y de ulna, siendo este el primer caso de iniencefalia reportado en Colombia. Además aportar criterios diagnósticos para la identificación de casos y hacer una revisión de la literatura publicada sobre este síndrome.

\section{REPORTE DE CASO}

Recién nacido de sexo femenino, madre de 14 años primigestante, ingresa a la institución de tercer nivel de complejidad, que atiende pacientes en su mayoría de la red pública del suroccidente colombiano, por inicio de actividad uterina y trabajo de parto pretérmino, con ecografía de las 26 semanas que sugiere malformación en columna toracolumbar y polihidramnios. Por progresión de cambios cervicales y presentación de cara, se realiza cesárea a las 27 semanas de gestación. Se obtuvo un recién nacido femenino, con peso de 975 g vivo, Apgar 1/10, el cual fallece antes de 5 minutos. Con consentimiento informado de los padres se le realizó autopsia fetal.

El resultado del examen anatomopatológico mostró: talla de $25 \mathrm{~cm}$, perímetro cefálico de 26 $\mathrm{cm}$, perímetro torácico de $24 \mathrm{~cm}$; hiperextensión del cuello que lleva a retroflexión forzada de la cabeza, con unión de la porción occipital a la región cervical y torácica de la columna, con continuidad de tejidos blandos, piel y cabello de la cabeza a la espalda. En el punto de la unión, se encontró estructura quística que se concluyó era un meningocele (figura 1); orejas de implantación baja, micrognatia (figura 2); en la radiografía se observó: retroflexión marcada de columna cervical, fusión de cuerpos vertebrales cervicales, hemivértebras torácicas e hipoplasia de: clavículas, ulnas y radios (figura 3).

Figura 1. Vista lateral. Se observa retroflexión forzada de la cabeza, produciendo unión de la porción occipital a la región cervical y torácica de la columna, además meningocele.

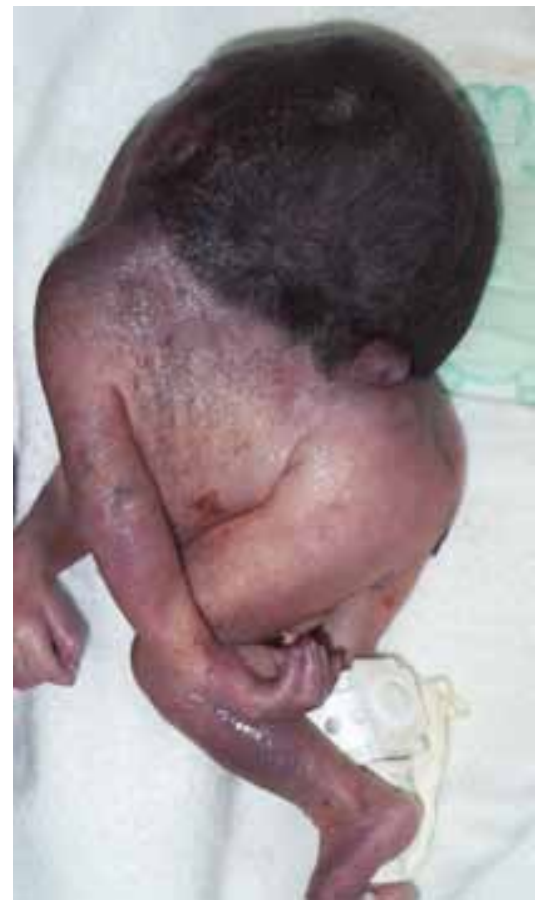


Figura 2. Vista frontal. Se observa hipoplasia del maxilar inferior y orejas de implantación baja.

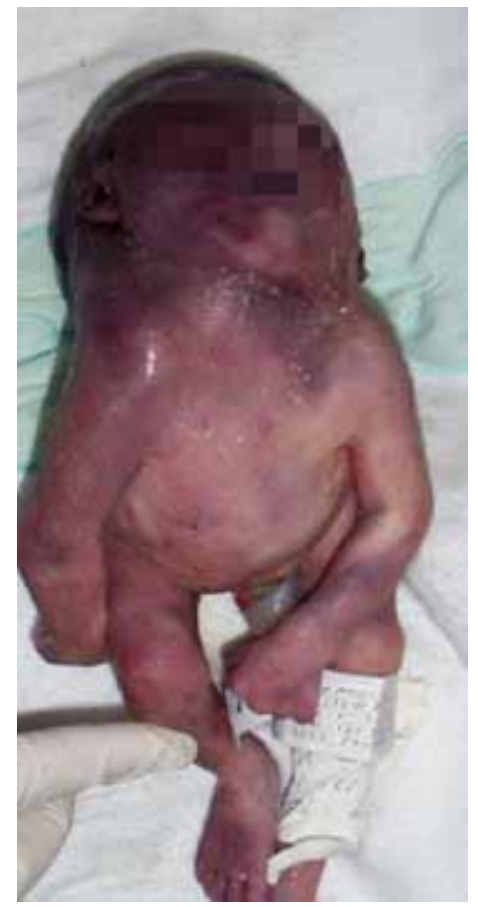

Figura 3. Rayos X vista lateral. Se observan las alteraciones vertebrales.

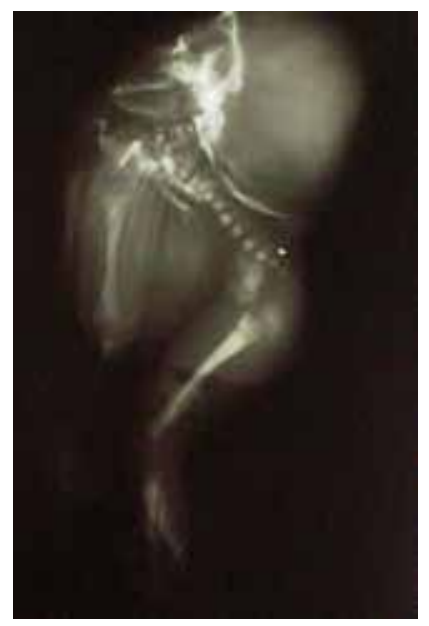

\section{MATERIALES Y MÉTODOS}

Se realizó una búsqueda en la bases de dato Medline vía PubMed con criterio de búsqueda avanzada, con la palabra clave iniencephaly en título y/o abstract. Bajo estas características se encontraron 110 artículos, de los cuales se revisaron 15, por su actualidad y relevancia. De estos, 3 fueron artículos de revisión y 13 , reportes de caso.

\section{DISCUSIÓN}

La iniencefalia es un defecto congénito poco frecuente, del cual existen solo reportes de caso que estiman la prevalencia, con uno o dos casos en períodos de tiempo de uno o dos años en una ciudad determinada. ${ }^{1,3}$ El Estudio Colaborativo Latinoamericano de Malformaciones Congénitas (ECLAMC) no reportó ningún caso entre 1982 y 2001, en 3.574.609 nacimientos ${ }^{5}$ y, según comunicación vía correo electrónico, el doctor Eduardo Castilla, coordinador del ECLAMC, afirma que después del 2001 y hasta el 1 de marzo de 2011, no se había reportado ningún caso de iniencefalia. En la literatura revisada en Colombia, se encontró que la prevalencia de defectos del tubo neural fue de 29 en 10000 en Cali y 9 en 10000 en Bogotá, entre 2004 y 2005. ${ }^{6}$ Otro estudio que incluyó 7 instituciones en tres ciudades y una vigilancia de 52744 nacimientos reportó una prevalencia de 9,9:10 000 entre 2001 y 2007. ${ }^{7}$ No reportaron algún caso de iniencefalia, por lo cual este es el primer caso reportado en Colombia.

El caso aquí expuesto corresponde a una iniencefalia cerrada, según la clasificación de Lewis, quien diferencia las presentaciones como: abierta (con encefalocele) y cerrada (sin encefalocele). ${ }^{4,8}$ Este caso no presentaba raquisquisis, entidad frecuente en los casos de iniencefalia. La iniencefalia es más frecuente en fetos femeninos, en una razón de 9 a 1;,4 puede también, estar asociada con múltiples anomalías congénitas: ${ }^{5}$ en una revisión de la literatura, Chen reporta 63 casos con diagnóstico prenatal de iniencefalia, de los cuales, el 75\% presentaba otras malformaciones asociadas, siendo la anencefalia la más frecuente; otros defectos asociados son onfalocele, encefalocele, hernia diafragmática, atresias intestinales, mielomeningocele, hidrocefalia, cardiopatías, holoprosencefalia, queilopalatosquisis y defectos renales. ${ }^{4}$ El caso aquí reportado presentó meningocele, hipoplasia de radio y ulnas, fusión y hemivértebras cervicotorácicas, siendo esta una presentación poco común. 
La fisiopatología de la iniencefalia no es clara pero puede producirse por defecto óseo en la porción escamosa del hueso occipital y vértebras cervicales y torácicas pobremente formadas; puede presentarse encefalocele, raquisquisis y meningocele. Con el foramen magno amplio se produce retroflexión marcada de la cabeza, lo que causa que quede permanentemente unida a la espalda y produzca continuidad de tejidos blandos subyacentes, debido a lo cual el defecto queda total o parcialmente recubierto por piel ${ }^{1,4}$

El diagnóstico prenatal de iniencefalia, al igual que el de todos los defectos congénitos, es de trascendental importancia para establecer el manejo y el pronóstico a fin de informar a los padres. ${ }^{4,9,10} \mathrm{El}$ defecto se origina en la cuarta semana del desarrollo embrionario y puede sospecharse en las ecografías rutinarias de control prenatal entre las 11 y 14 semanas o entre las 20 y 24 semanas y su confirmación se da por medio de ecografía de detalle anatómico. Además, la relación entre altos niveles séricos maternos de alfafeto proteína, y la presencia de defectos del tubo neural se manifiesta en la niencefalia. ${ }^{2,11}$ En los casos de iniencefalia es conveniente la realización de cariotipo con bandas $\mathrm{G}$ de alta resolución, de muestra obtenida por biopsia de vellosidad coriónica, amniocentesis, cordocentesis, o en el recién nacido, para descartar alteraciones cromosómicas numéricas o estructurales. El diagnóstico prenatal de iniencefalia y anomalías asociadas establecería el pronóstico del feto, daría la posibilidad de la interrupción temprana del embarazo, ${ }^{2,4,5}$ permitiría la preparación temprana del equipo médico para la atención de la madre, el parto y el feto, con el fin de evitar una cesárea. Este diagnóstico temprano también ayudaría a concientizar a la familia sobre la importancia de la realización de autopsia fetal que incluya radiografías, como las realizadas en este caso, y resonancia magnética para delimitar los defectos óseos. ${ }^{12,13}$

\section{CONCLUSIÓN}

Este reporte suma un nuevo caso de iniencefalia asociado con meningocele, estudiado con autopsia fetal y radiografías, a la literatura mundial; y es el primero reportado en Colombia.

\section{CONSIDERACIONES ÉTICAS}

La autopsia, el reporte del caso y la publicación de las fotografías se hacen con la firma del consentimiento informado de los padres. En ningún momento se vulneró la confidencialidad de las identidades del feto y su madre.

\section{AGRADECIMIENTOS}

A Verónika Ceballos Núñez, estudiante de Biología de la Universidad del Valle, por su apoyo en la búsqueda bibliográfica, escritura y, traducción del informe final; al Doctor Eduardo Castilla, Coordinador del ECLAMC por compartir sus conocimientos, y la información de los registros de la base de datos del ECLAMC.

\section{REFERENCIAS}

1. Kulaylat NA, Narchi H. Iniencephaly: an uncommon neural tube defect. J Pediatr 2000;136:414.

2. Tejerizo A, de Marino M, Belloso M, Villalba A, Gonzalez MA, Ruiz A, et al. Iniencefalia. Clin Invest Gin Obst 2006;33:130-9.

3. Aguila A, Nazer J, Bentjerodt R. Prevención de defectos de cierre del tubo neural, a propósito de un caso de iniencefalia. Rev Chil Obstet Ginecol 1994;59:53-6

4. Chih-Ping Chen. Prenatal diagnosis of iniencephaly. Taiwan J Obstet Gynecol 2007;46:199-208.

5. Castilla E, Orioli IM. ECLAMC: The Latin-American Collaborative Study of Congenital Malformation. Community Genet 2004;7:76-94.

6. Saldarriaga W, Blanco-Tamayo G, Bravo-López D, Díaz-Hung A, Fandiño A, Isaza C. La altitud como factor de riesgo para defectos del tubo neural. Rev Colomb Obstet Ginecol 2007;58:189-93.

7. Zarante I, Franco L, López C, Fernández N. Frecuencia de malformaciones congénitas: evaluación y pronóstico de 52.744 nacimientos en tres ciudades colombianas. Biomedica 2010;30:65-71.

8. Sahid S, Sepulveda W, Dezerega V, Gutierrez J, Rodriguez L, Corral E. Iniencephaly: prenatal diagnosis and management. Prenat Diagn 2000;20:202-5. 
9. Sepulveda W, Wong AE, Fauchon DE. Fetal spinal anomalies in a first-trimester sonographic screening program for aneuploidy. Prenat Diagn 2011;31:107-14.

10. Gadodia A, Gupta P, Sharma R, Kumar S, Gupta G. Antenatal Sonography and MRI of Iniencephaly apertus and clausus. Fetal Diagn Ther 2010;27:178-80.

11. Dashe JS, Twickler DM, Santos-Ramos R, McIntire DD, Ramus R. Alpha-fetoprotein detection of neural tube defects and the impact of standard ultrasound. Am J Obstet Gynecol 2006;195:1623-8.
12. Joó JG, Beke A, Papp C, Szigeti Z, Csaba A, Papp Z. Major diagnostic and pathological features of iniencephaly based on twenty-four cases. Fetal Diagn Ther 2008;24:1-6.

13. Pungavkar SA, Sainani NI, Karnik AS, Mohanty PH, Lawande MA, Patkar DP, et al. Antenatal diagnosis of iniencephaly: sonographic and MR correlation: a case report. Korean J Radiol 2007;8:351-5. 